\title{
Development and implementation of "Check of Medication Appropriateness" (CMA): advanced pharmacotherapy-related clinical rules to support medication surveillance
}

Charlotte Quintens $s^{1,2^{*}+}$ (D), Thomas De Rijdt ${ }^{1,2+}$, Tine Van Nieuwenhuyse ${ }^{2}$, Steven Simoens ${ }^{1}$, Willy E. Peetermans ${ }^{3,4}$, Bart Van den Bosch ${ }^{5,6}$, Minne Casteels ${ }^{1}$ and Isabel Spriet ${ }^{1,2}$

\begin{abstract}
Background: To improve medication surveillance and provide pharmacotherapeutic support in University Hospitals Leuven, a back-office clinical service, called "Check of Medication Appropriateness" (CMA), was developed, consisting of clinical rule based screening for medication inappropriateness. The aim of this study is twofold: 1) describing the development of CMA and 2) evaluating the preliminary results, more specifically the number of clinical rule alerts, number of actions on the alerts and acceptance rate by physicians.

Methods: CMA focuses on patients at risk for potentially inappropriate medication and involves the daily checking by a pharmacist of high-risk prescriptions generated by advanced clinical rules integrating patient specific characteristics with details on medication. Pharmacists' actions are performed by adding an electronic note in the patients' medical record or by contacting the physician by phone. A retrospective observational study was performed to evaluate the primary outcomes during an 18-month study period.

Results: 39,481 clinical rule alerts were checked by pharmacists for which 2568 (7\%) electronic notes were sent and 637 (1.6\%) phone calls were performed. 37,782 (96\%) alerts were checked within four pharmacotherapeutic categories: drug use in renal insufficiency (25\%), QTc interval prolonging drugs (11\%), drugs with a restricted indication or dosing (14\%) and overruled very severe drug-drug interactions (50\%). The emergency department was a frequently involved ward and anticoagulants are the drug class for which actions are most frequently carried out. From the 458 actions performed for the four abovementioned categories, $69 \%$ were accepted by physicians.
\end{abstract}

Conclusions: These results demonstrate the added value of CMA to support medication surveillance in synergy with already integrated basic clinical decision support and bedside clinical pharmacy. Otherwise, the study also highlighted a number of limitations, allowing improvement of the service.

Keywords: Check of medication appropriateness (CMA), Clinical validation of prescriptions, Clinical decision support (CDSS), Computerized physician order entry (CPOE), Medication surveillance, Clinical rules

\footnotetext{
* Correspondence: charlotte.quintens@uzleuven.be

${ }^{\dagger}$ Charlotte Quintens and Thomas De Rijdt contributed equally to this work.

'Department of Pharmaceutical and Pharmacological Sciences, KU Leuven,

Herestraat 49, B-3000 Leuven, Belgium

${ }^{2}$ Pharmacy Department, University Hospitals Leuven, Herestraat 49, B-3000

Leuven, Belgium

Full list of author information is available at the end of the article
}

(c) The Author(s). 2019 Open Access This article is distributed under the terms of the Creative Commons Attribution 4.0 International License (http://creativecommons.org/licenses/by/4.0/), which permits unrestricted use, distribution, and reproduction in any medium, provided you give appropriate credit to the original author(s) and the source, provide a link to the Creative Commons license, and indicate if changes were made. The Creative Commons Public Domain Dedication waiver (http://creativecommons.org/publicdomain/zero/1.0/) applies to the data made available in this article, unless otherwise stated. 


\section{Background}

Inappropriate prescribing has been shown to be an independent risk factor for adverse drug events (ADE) and several studies reported that ADEs can cause hospitalization [1]. Moreover, half of these hospital admissions are preventable [2, 3]. Different strategies were already described to prevent inappropriate prescribing, i.e. 1) a clinical pharmacist attending physician rounds, 2) computerized physician order entry (CPOE) and 3) CPOE with clinical decision support system (CDSS) [4].

In order to augment the safety and quality of patients' therapy, front-office bedside clinical pharmacy services were set up in many European countries, after these were implemented in the UK, Canada and the USA since the '70s [5]. Bedside, embedded clinical pharmacists are typically involved in medication reconciliation and review, medication counselling at discharge and targeted projects improving medication use [6]. However, due to limited healthcare budget, which is the case in many European countries, bedside clinical pharmacy services are not implemented on a hospital-wide basis but restricted to high-risk patient populations such as geriatric patients characterized by polypharmacy, critically ill patients or patients admitted at the emergency department or on surgical wards with rapid patient turnover $[5,6]$.

With the increase in information and implementation of CPOE, medical and treatment data are available in a structured way which led rapidly to embedding basic and advanced CDSS in the CPOE (CPOE/CDSS) in order to support appropriate prescribing [7-9]. During the prescription process, basic CDSS analyse the data in the CPOE based on clinical rules and provide alerts automatically signalling clinical problems such as drug-drug interactions (DDI), basic dosing guidance, drug-allergy checking, duplicate therapy, etc. Nevertheless, basic CDSS is only providing support at the step of prescribing and not during follow-up treatment, without taking into account relevant biochemical parameters [9]. More advanced CDSS also contain clinical rules combining several sources of information on the characteristics of individual patients such as laboratory values [9-13].

Studies have shown a clear benefit of CPOE/CDSS with a significant decrease in prescription errors and ADEs [7, 14-18]. Additionally, Rommers et al. showed the added value of advanced clinical rules in preventing ADEs when used in combination with basic CDSS [10]. Conversely, the rise in health information technology has induced new pitfalls such as 'alert fatigue', i.e. the ignorance of both relevant and non-relevant alerts by health professionals because of moderate to low clinical relevance of some alerts, especially for basic clinical rules [18-20]. Eppenga et al. demonstrated the improved (but still not optimal) clinical relevance of medication alerts when including more patient-related characteristics [13].
Furthermore, sophisticated CDSS requires well-established digital communication, an effective integration of data and frequent updates [19].

In the University Hospitals Leuven, Belgium, medication surveillance is supported by both bedside clinical pharmacists on high-risk wards (geriatrics, pediatrics, emergency department, trauma surgery, abdominal surgery and septic orthopedic surgery) and a basic CPOE/CDSS. Given the limitations of the basic CDSS (i.e. alert fatigue; no integration of biochemical parameters) and the limited implementation of bedside clinical pharmacy services along with the aim for medication surveillance and review on a hospital-wide basis, which is driven by the hospital accreditation standards, University Hospitals Leuven has developed a new back-office clinical service: the "Check of Medication Appropriateness" (CMA).

This service consists of advanced clinical rule alerts aiming exclusively at hospital pharmacists, followed by pharmacists' performed actions aiming at physicians in case of medication inappropriateness. The objective of this manuscript is to describe the development of CMA and to evaluate the preliminary results of this new clinical service.

\section{Methods}

\section{Setting}

The University Hospitals Leuven is a 1995-bed academic tertiary care centre. The Hospital's Information System (HIS) with patients' electronic medical records for noncritically ill patients integrates patient-specific data (including demographics, laboratory values, clinical parameters), surgical and radiology reports, physicians' and nursing notes, CPOE and medication orders. The CPOE system is integrated with a CDSS, which provides support at the moment of prescribing by checking for DDIs, drug-food interactions, drug use during pregnancy and lactation, allergies, maximum dosage and therapeutic duplication. The safety alerts for DDIs are based on a national database (DelphiCare ${ }^{\oplus}, \mathrm{APB}$, Belgium). DDIs are categorized in three groups: very severe DDIs, severe DDIs and other DDIs (with minor clinical relevance). The prescribing physician only has to overrule the alerts for very severe DDIs, and if so, should provide a motivation. Prescribing for patients admitted on the intensive care unit (ICU) is carried out in another software system without integrated CDSS.

\section{Set-up of CMA service}

The objective of CMA is to validate the treatment of patients at risk for potentially inappropriate medication (PIM), including drug-related problems (DRPs) and ADEs by combining structured data available from HIS and by using standardized algorithms, also referred to as clinical rules. The development process (realized with 0.2 full-time 
equivalents (FTE) pharmacist) consists of three phases: (1) development of the clinical rules, (2) development of the CMA, and (3) validation of the service.

\section{Development of the clinical rules}

The need for specific clinical rules was determined by a multi-method approach. Patients at risk for PIMs were identified based on bedside clinical pharmacy experience and patient safety incident reports. The clinical rules were defined based on literature and (inter)national guidelines (e.g. IDSA guideline for the management of catheter-related infections [21], EHRA guideline on the use of new oral anticoagulants [22], etc.). All clinical rules were reviewed in a multidisciplinary team consisting of clinical pharmacists and medical staff, and approved by the hospital board and Pharmacy \& Therapeutics committee (P\&T) to finally result in a definite set of 78 advanced clinical rules which were considered to be relevant for our hospital practice (Additional file 1). These clinical rules were grouped in five pharmacotherapeutic categories i.e. 1) overrules of alerts for very severe DDIs generated by the CDSS, 2) drugs with a restricted indication or dosing, 3) medication potentially leading to biochemical changes, 4) potential sequential therapy (intravenous (IV) to oral switch), and 5) others. The third category, i.e. medication use potentially leading to biochemical changes, is further divided in six subcategories as shown in Table 1.

\section{Development of the CMA}

The algorithms are formulated as 'if-then' rules and the CMA system, based on a Microsoft Access database, generates alerts invoked by specific triggers ('clinical rule alert criteria') based on screening the available data in the electronic medical record and CPOE. In this way, medical records along with treatment schemes are screened, both in non-critically ill hospitalized patients and patients admitted at the day care hospital. Screening and generation of the alerts take place daily at $12 \mathrm{am}$.

For the category 'potential sequential therapy', the algorithm is automatically selecting patients with presumed intact gastrointestinal absorption and a prescription for an IV bio-equivalent drug. An electronic note explaining the potential IV to oral switch is automatically provided in the patient's medical file. For the other four categories, the results of the screening, the clinical rule alerts, are listed on a structured worklist. This worklist is assessed on a daily basis ( 0.5 FTE) by a trained hospital pharmacist for appropriateness of treatment. For each clinical rule, a user-friendly standardized flowchart or decision tree was drawn, on which the hospital pharmacist can rely while validating the prescriptions. When deemed necessary, an action on the alert is carried out by leaving an electronic note for the treating physician in the patient's medical record. The message of the note is predefined for each clinical rule in the concerning flowchart. In case of a potentially severe $\mathrm{ADE}$, the physician is also contacted by phone next to the electronic note.

\section{Validation of the CMA}

To ensure that the system selects the patients at risk for predefined PIMs, a validation was performed by two different methods. First, fictive patients were used to create fictive medication orders in the CPOE to test if the medication orders are screened appropriately based on the predefined algorithms. Through the use of a set of test patients, the sensitivity of the system can be evaluated. Second, the CMA was performed behind-the-scene on

Table 1 Pharmacotherapeutic categories and subcategories used to define the clinical rules ${ }^{a}$

\begin{tabular}{|c|c|c|}
\hline & Category (and subcategories) & Example of a clinical rule \\
\hline 1 & Overrules of alerts for very severe DDIs generated by the CDSS & $\begin{array}{l}\text { Reduced effect of valproic acid by carbapenems leading to an increased } \\
\text { risk of convulsions }\end{array}$ \\
\hline 2 & Drugs with a restricted indication or dosing & Patient with high dose meropenem \\
\hline \multirow[t]{7}{*}{3} & Medication use potentially leading to biochemical changes & \\
\hline & * Drug use in renal insufficiency & Patient with a $\mathrm{CrCl}<30 \mathrm{ml} / \mathrm{min}$ and treated with metformin \\
\hline & * Drugs with high potential of QTc interval prolongation & Patient with a QTc $>450 / 470 \mathrm{~ms}$ and treated with haloperidol \\
\hline & * Drug use associated with hyperpotassemia & Patient with a $\mathrm{K}>5.5 \mathrm{mmol} / \mathrm{L}$ and treated with an ACE inhibitor \\
\hline & * Drug use associated with hypopotassemia & $\begin{array}{l}\text { Patient with a } K<3.5 \mathrm{mmol} / \mathrm{L} \text { and treated with flucloxacillin without } \\
\text { potassium supplementation }\end{array}$ \\
\hline & ${ }^{*}$ Drug use associated with supratherapeutic INR & Patient with a supratherapeutic INR $(I N R>4)$ and treated with a VKA \\
\hline & ${ }^{*}$ Drug use associated with bone marrow suppression & $\begin{array}{l}\text { Patient with an absolute neutrophil count }<1.5^{*} 10^{9} / \mathrm{L} \text { and treated with } \\
\text { clozapine }\end{array}$ \\
\hline 4 & Potential sequential therapy for bio-equivalent drugs & Potential sequential therapy for levofloxacine \\
\hline 5 & Others & $\begin{array}{l}\text { Patient treated with non-crushable drugs administered through enteral } \\
\text { feeding tube }\end{array}$ \\
\hline
\end{tabular}

${ }^{a} D D I$ drug-drug interaction, $C D S S$ clinical decision support system, INR international normalized ratio, $\mathrm{CrCl}$ creatinine clearance, $A C E$ angiotensin converting enzyme, VKA vitamin $\mathrm{K}$ antagonist 
hospitalized patients. The medical record of the patients who were listed on the worklist was checked manually to search for false positive results to evaluate the specificity of the system. Based on this validation, the structure of the clinical rules was adapted and optimized to reduce the number of false positive and false negative results.

Twenty-one hospital pharmacists are participating in the CMA service. To assess if the validation is carried out in the same way, interrater reliability using kappa statistics was determined. The kappa ( $\mathrm{k}$ ) value, a chance-corrected index of agreement, can range from -1 (complete disagreement) to +1 (perfect agreement). The trained hospital pharmacists had to check ten clinical rule alerts, using the available flowcharts. An average $\mathrm{k}$ of 0.79 was obtained, which corresponds to an adequate agreement in performing the checking of PIMs based on the standardized flowcharts.

\section{Study design}

The impact of CMA was evaluated in a retrospective observational study during an 18-month period, i.e. from March 2016 up to August 2017. All non-critically ill hospitalized patients and patients admitted at the day care hospital during the study period were included in this analysis.

\section{Quantitative evaluation of preliminary results}

A quantitative evaluation was performed by documenting the number of prescriptions that were checked by the hospital pharmacist (i.e. the number of clinical rule alerts) and the number of actions on alerts. Alerts were considered as clinically relevant whenever an action (electronic note with or without a phone call) was performed by the pharmacist. The analysis was also carried out without taking into account the automatic notes for potential sequential therapy.

The same evaluation was done for the following specific pharmacotherapeutic categories: drug use in renal insufficiency, drugs with high potential of QTc interval prolongation, drugs with a restricted indication or dosing and overruled very severe DDIs.

\section{Qualitative evaluation of preliminary results}

For the abovementioned four specific categories, patients' mean age, the top five of the involved wards and the top five of the involved drugs were evaluated, for both the actions for which electronic notes vs. electronic notes plus phone calls were carried out. The age and wards were assessed in order to get an idea whether these patient populations are different from the ones for which we have bedside clinical pharmacy services.

\section{Acceptance rate}

An evaluation of the acceptance rate by the treating physician was performed. 300 unique patients for which an automatic note for sequential therapy was sent, 300 unique patients for which the pharmacist had put a note in the patient's file for four other categories (drug use in renal insufficiency, drugs with high potential of QTc interval prolongation, drugs with a restricted indication or dosing and overruled very severe DDIs) and 300 unique patients for which the pharmacist had called the treating physician in combination with leaving an electronic note for the same four categories, were randomly selected during the 18-month period. Patients' electronic medical records were explored retrospectively to evaluate whether the pharmacists' advice had been followed. Acceptance was defined as a modification of a prescription (stop or dose correction) or a further follow-up of clinical and/or laboratory parameters in function of the specific flowchart within $72 \mathrm{~h}$ after the pharmacist's action. Reasons for non-compliance with the pharmacist's advice were not investigated. This evaluation was primarily carried out by one pharmacist (CQ). In case of doubt, a meeting was organized to reach consensus together with a second senior hospital pharmacist (IS) in order to take the final decision.

\section{Statistical analysis}

For this evaluation only descriptive analyses were performed.

\section{Results}

\section{Quantitative evaluation of preliminary results}

During the 18-month study period, 92,050 clinical rule alerts were extracted for which 24,943 (27\%) electronic notes were sent and 637 (0.7\%) electronic notes supplemented by phone calls were carried out. When analysed without the automatic warnings for sequential therapy, 39,481 clinical rule alerts were checked for which 2568 (7\%) electronic notes were sent and 637 (1.6\%) electronic notes plus phone calls were carried out (Fig. 1).

Table 2 shows the results for the four specific pharmacotherapeutic categories that were analysed in more detail. In total, 37,782 checks were carried out for these preselected categories, counting for $96 \%$ of the clinical rule alerts without taking into account the automatic notes for potential sequential therapy (Fig. 1; Table 2).

\section{Qualitative evaluation of preliminary results}

Table 3 shows that for the four preselected categories the mean age of patients for which an electronic note was sent, was between 46.7 up to 74.2 years of age. Actions were frequently carried out for patients admitted on three wards, i.e. the emergency department, vascular and cardiac surgery. When looking into the drugs for which notes or phone calls were made, anticoagulants are frequently involved (cf. Table 3). In the evaluation of drugs used in renal insufficiency, 57\% of electronic notes were sent for direct oral anticoagulants (DOACs) (10\%) and low molecular weight heparins (LMWHs) $(47 \%)$. Of the phone calls in this category, $73 \%$ were carried out 


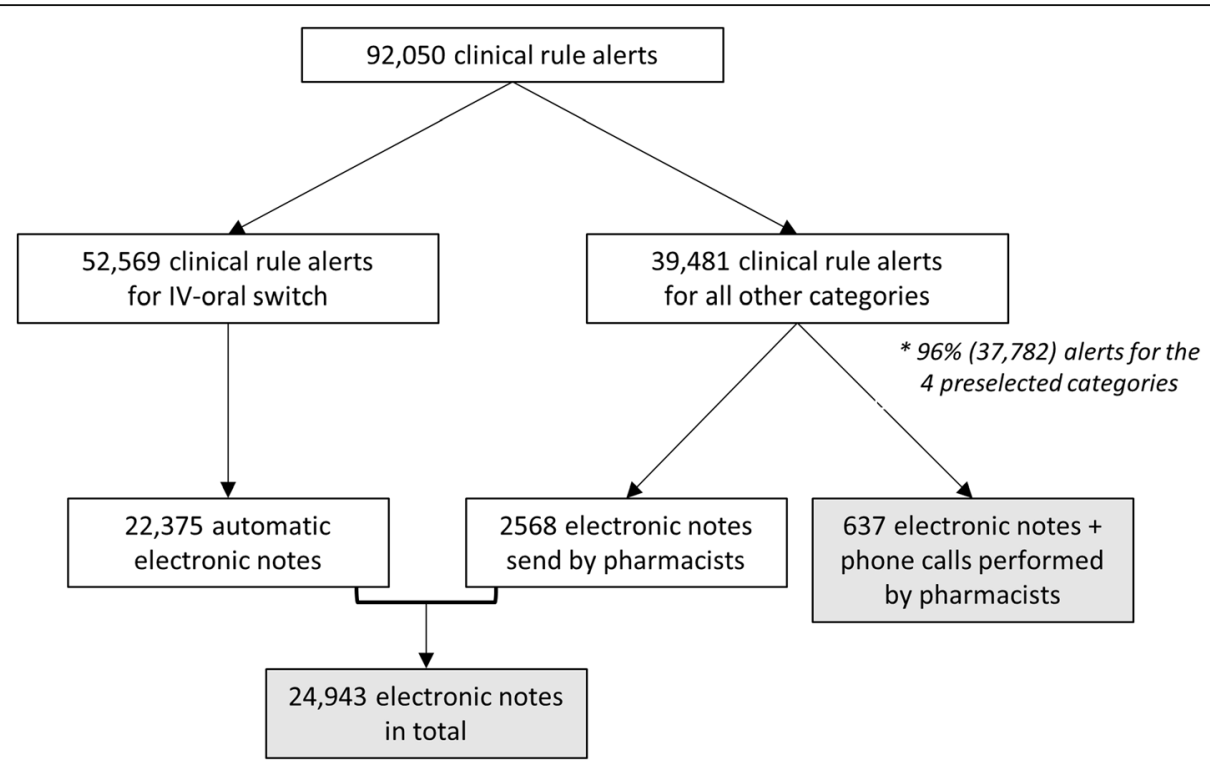

Fig. 1 Flow diagram of the quantitative evaluation. *4 preselected categories: drug use in renal insufficiency, drugs with high potential of QTC interval prolongation, drugs with a restricted indication or dosing and overruled very severe DDIs

for anticoagulants of which $21 \%$ was carried out for DOACs and $54 \%$ for LMWHs (total percentages not shown in Table 3). When focusing on the evaluation of overruled DDIs, the overruled combination of DOACs with other anticoagulants (often LMWHs) was the most frequent alert for which a phone call was carried out $(37 \%)$ or an electronic note was sent $(15 \%)$. The evaluation of the actions performed for the category of drugs with a restricted indication or dosing, revealed a high percentage of recommendations carried out for high dose meropenem (2 g, q8h).

\section{Acceptance rate}

The sample of 300 unique patients for which an electronic alert was sent for sequential therapy resulted finally in 341 actions, as in some patients there was more than one bio-equivalent drug. Of these 341 actions, 112 (33\%) led to a switch from IV to oral therapy. For 64 (19\%) of the electronic notes, the IV drug was stopped and for 165 $(48 \%)$ of the notes, the advice was not accepted.
The sample of 300 unique patients for which the pharmacist left an electronic note in the patient's file for the four preselected categories, resulted finally in 229 actions after excluding duplicates and notes for which the acceptance rate could not be verified because of a discharge or transfer to another hospital unit. Among the 229 pharmacy notes, 129 (56\%) were accepted by the physician.

The sample of 300 unique patients for which the pharmacist had called the treating physician in combination with leaving an electronic note in the patient's file for the same four categories, resulted also in 229 actions after the same exclusion. Among the 229 pharmacy phone calls, 189 (83\%) were accepted by the physician.

Tables 4 and 5 shows the acceptance rate of notes and notes supplemented by phone calls for each category. In total, $318(69 \%)$ out of 458 actions led to a documented modification in therapy or follow-up of clinical and/or laboratory parameters.

Table 2 Number of checks and actions during the 18-month study period for four pharmacotherapeutic categories ${ }^{b}$

\begin{tabular}{llll}
\hline Category & $\begin{array}{l}\text { Number of prescriptions } \\
\text { checked }(\mathrm{n})\end{array}$ & $\begin{array}{l}\text { Number of electronic notes } \\
(\mathrm{n}(\%))\end{array}$ & $\begin{array}{l}\text { Number of electronic notes }+ \\
\text { phone calls }(\mathrm{n}(\%))\end{array}$ \\
\hline Drug use in renal insufficiency & 9381 & $444(4.7 \%)$ & $81(0.9 \%)$ \\
Drugs with high potential of QTc prolongation & 4223 & $608(14 \%)$ & $139(3.3 \%)$ \\
Drugs with restricted indication or dosing & 5276 & $448(9 \%)$ & $142(2.7 \%)$ \\
Overruled very severe DDIs & 18,902 & $939(5 \%)$ & $259(1.4 \%)$ \\
Total & 37,782 & $2439(7 \%)$ & $621(1.6 \%)$
\end{tabular}

${ }^{\mathrm{b}} \mathrm{DD} /$ drug-drug interaction, $n$ absolute number 


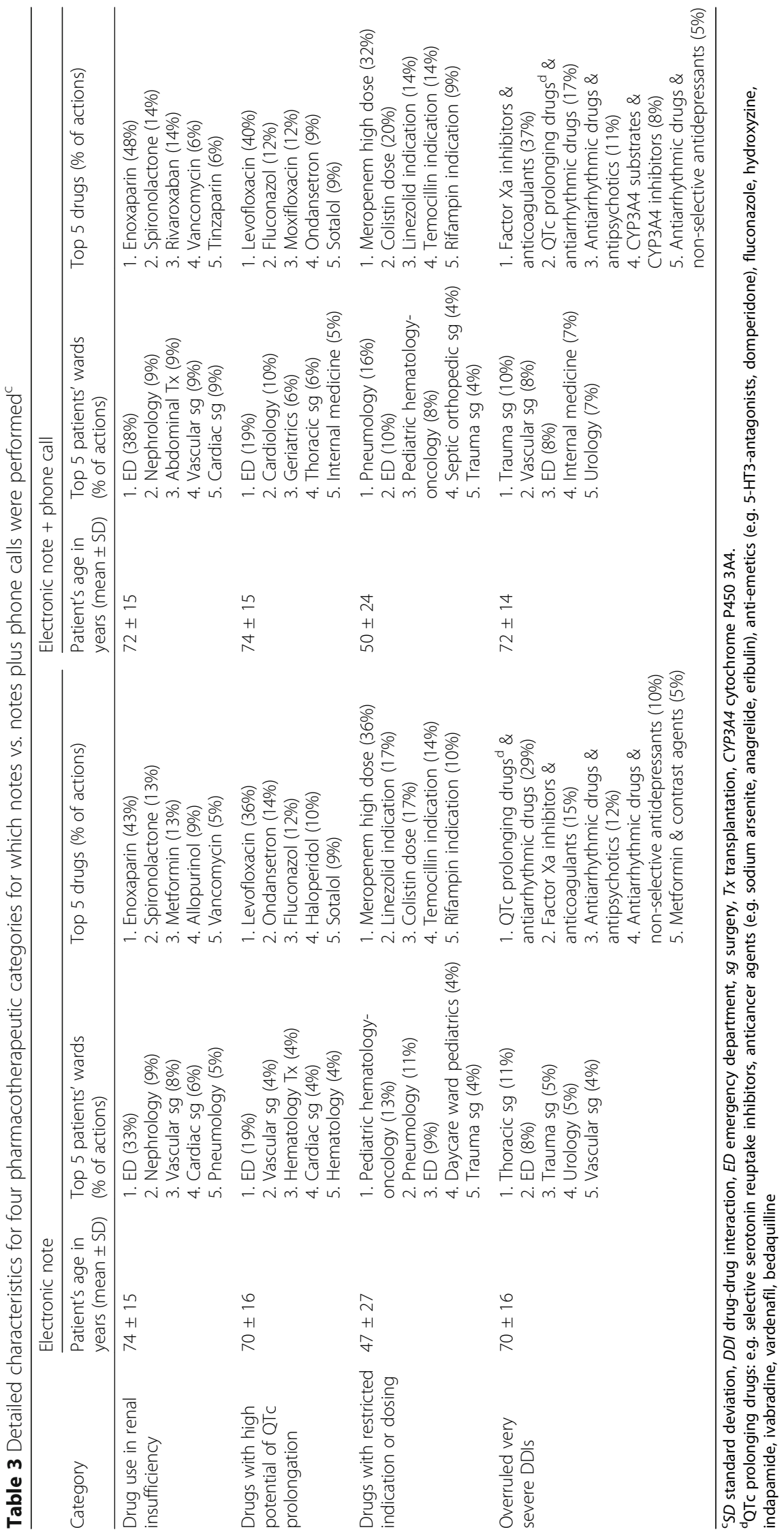


Table 4 Acceptance rate of actions for four pharmacotherapeutic categories performed by electronic notes ${ }^{e}$

\begin{tabular}{|c|c|c|c|c|c|}
\hline \multirow[t]{2}{*}{ Category } & \multirow{2}{*}{$\begin{array}{l}\text { Total number } \\
\text { of patients (n) }\end{array}$} & \multicolumn{2}{|l|}{ Exclusion } & \multirow{2}{*}{$\begin{array}{l}\text { Total number } \\
\text { of actions (n) }\end{array}$} & \multirow{2}{*}{$\begin{array}{l}\text { Number of actions } \\
\text { accepted by physician } \\
(\mathrm{n}(\%))\end{array}$} \\
\hline & & Duplicates & $\begin{array}{l}\text { Not possible } \\
\text { to verify* }\end{array}$ & & \\
\hline Drug use in renal insufficiency & 61 & 10 & 6 & 45 & $31(69 \%)$ \\
\hline Drugs with high potential of QTc prolongation & 78 & 4 & 11 & 63 & $34(54 \%)$ \\
\hline Drugs with restricted indication or dosing & 51 & 3 & 5 & 43 & $21(49 \%)$ \\
\hline Overruled very severe DDIs & 110 & 25 & 7 & 78 & $43(55 \%)$ \\
\hline Total & 300 & 71 & & 229 & $129(56 \%)$ \\
\hline
\end{tabular}

${ }^{\mathrm{e}} \mathrm{DDI}$ drug-drug interaction, $n$ absolute number. ${ }^{*}$ notes for which the acceptance rate could not be verified because of a discharge or transfer to another hospital unit

\section{Discussion}

During the study period, a back-office CMA service, embedding 0.5 FTE hospital pharmacists, yielded 24,943 electronic notes and 637 electronic notes supplemented by phone calls, concerning potentially very harmful ADEs or DRPs. When analysed without the automatic warnings for sequential therapy, $96 \%$ of the checks were performed in four specific pharmacotherapeutic categories: drug use in renal insufficiency, drugs with high potential of QTc interval prolongation, drugs with a restricted indication or dosing and overruled very severe DDIs. Acceptance rate of pharmacy notes and pharmacy notes supplemented by phone calls, carried out in the abovementioned categories, was $56 \%$ and $83 \%$, respectively.

As expected, geriatric patients ( $>75$ years) and children ( $<18$ years) were only rarely included, since bedside clinical pharmacy services are already provided on a daily base at both wards. Patients admitted at emergency and cardiac surgery departments were most frequently involved. These results contribute to a risk based assessment in order to prioritize future investments in bedside clinical pharmacy services at these specific wards. Anticoagulants, mentioned in 6 of the 78 clinical rules, are a major drug class for which advices were formulated. These results suggest that current knowledge on the potential dangers of (the novel oral) anticoagulants, when used in patients with decreased renal function or when used in combination with other anticoagulants, is lacking. These results indicate that it is worthwhile to look for potential ADEs with these agents. Concerning other issues revealed by CMA, action was already undertaken e.g. for the use of meropenem high dose $(2 \mathrm{~g}, \mathrm{q} 8 \mathrm{~h})$ a newsletter was sent to the pediatric hematology-oncology ward.

Our results indicate that the implementation of CMA is a significant addition to the standard services provided by the currently implemented basic CDSS, which is running on a hospital wide basis but only supporting at the step of prescribing without taking into account relevant laboratory values, and also to bedside clinical pharmacy services, which provides support at any time of the treatment but only runs for a limited and highly selected patient population. CMA, which combines automated screening of multiple data sources in the patient's electronic medical record with the back-office evaluation of the patient's therapy by a trained hospital pharmacist, has the ability to provide pharmacotherapeutic support on a hospital wide basis. With maximum integration of patient specific characteristics with details on drug treatment, a personalized advice can be given. The organization of our CMA service was well considered before implementation and is based on algorithms, which rely on evidence-based literature and practice-based experience, user-friendly flowcharts and uniform advices which need approval by the P\&T committee before implementation. The obtained kappa value (0.79) also proves that there is a homogeneity between the pharmacists in checking the high risk prescriptions.

There are a lot of studies describing the implementation and evaluation of CPOE, basic and advanced CPOE/CDSS, but there are few studies [10, 23-26] describing services like the CMA, consisting of alerts aiming exclusively at

Table 5 Acceptance rate of actions for four pharmacotherapeutic categories performed by electronic notes plus phone calls ${ }^{f}$

\begin{tabular}{|c|c|c|c|c|c|}
\hline \multirow[t]{2}{*}{ Category } & \multirow{2}{*}{$\begin{array}{l}\text { Total number } \\
\text { of patients (n) }\end{array}$} & \multicolumn{2}{|l|}{ Exclusion } & \multirow{2}{*}{$\begin{array}{l}\text { Total number } \\
\text { of actions (n) }\end{array}$} & \multirow{2}{*}{$\begin{array}{l}\text { Number of actions } \\
\text { accepted by physician } \\
(\mathrm{n}(\%))\end{array}$} \\
\hline & & Duplicates & $\begin{array}{l}\text { Not possible } \\
\text { to verify* }\end{array}$ & & \\
\hline Drug use in renal insufficiency & 27 & / & 5 & 22 & $16(73 \%)$ \\
\hline Drugs with high potential of QTc prolongation & 66 & / & 3 & 63 & $48(76 \%)$ \\
\hline Drugs with restricted indication or dosing & 66 & / & 6 & 60 & $44(73 \%)$ \\
\hline Overruled very severe DDIs & 141 & 46 & 11 & 84 & $81(96 \%)$ \\
\hline Total & 300 & 71 & & 229 & 189 (83\%) \\
\hline
\end{tabular}

$\overline{\mathrm{f}} D D /$ drug-drug interaction, $n$ absolute number. ${ }^{*}$ notes for which the acceptance rate could not be verified because of a discharge or transfer to another hospital unit 
pharmacists, followed by pharmacists' performed actions in case of medication inappropriateness. Our CMA system is very similar to the pharmacy adverse drug event alerting system (ADEAS) developed by Rommers et al. [10] They formulated a comparable list of clinical rules $(n=121)$ divided in risk categories. Like CMA, the generated alerts go to the pharmacist and not directly to the prescribing physician to prevent alert fatigue with the physician. They use ADEAS as a tool for the hospital pharmacist for more clinical ward-based activities, whilst CMA is developed as a back-office clinical service [10]. In our opinion, a pharmacy decision support system like CMA and ADEAS is an essential added value to support medication surveillance and pharmacotherapy in European countries in which healthcare budget supporting bedside clinical pharmacy is scarce. Since clinical rules generally rely on literature-based evidence and practice-based evidence, we believe that the implementation of a similar service is possible in other European centers.

Our current adoption of the CMA service still demonstrates some limitations. First, when looking into the results without the automated suggestions for sequential therapy, the ratio of actions $(n=3205)$ to the total number of clinical rule alerts $(n=39,481)$ is low. Only $8 \%$ of the alerts were considered clinically relevant by the hospital pharmacists, which implies a large number of false positive alerts and unnecessary checks by the hospital pharmacist. This number of irrelevant alerts seems high, but a comparable percentage has also been observed in some previous studies which investigated one or more specific advanced medication alerts [12, 13, 23-25]. In the study of Rommers et al., a similar percentage (7.8\%) of the alerts resulted in advice to prevent possible ADEs [23]. The reasons why clinical rule alerts were classified as not relevant by the hospital pharmacist were not systematically analysed in our study. However some reasons for non-relevance were frequently identified: 1) the dosage was already adjusted, 2) the drug was (temporarily) stopped, 3) the monitored laboratory value or clinical parameter was already reverted to within the reference limits, 4) repeated alerts which were already evaluated by the pharmacist and 5) double alerts with the same content. The same reasons were already mentioned by de Wit et al. and Rommers et al. [12, 23] In the near future we plan to increase specificity of the CMA by further reformulating and fine-tuning the alert criteria of the clinical rules (e.g. by identifying and integrating more patient characteristics or parameters like weight, specific dose regimens, clinical symptoms etc.). Unfortunately, the inclusion of more alert criteria is limited because some patient characteristics are not structurally electronically documented in the medical record. So further automation is dependent on the digitalization of patients' characteristics, which was already mentioned by
Eppenga et al. [13] To prevent repeated alerts, the alert could be suppressed until a relevant change of one of the alert criteria makes the alert reappear. Already reverted laboratory values could be a result of delay in data delivery, which can be resolved by integration of the CMA in HIS resulting in a direct link with the laboratory system. Furthermore, we also have to reconsider the basic CDSS for DDIs. The limited number of actions carried out for overruled DDIs indicates that many of these DDIs have to be overruled by the physician without a high risk for the patient. These unnecessary overrules might definitely lead to frustration and alert fatigue for the prescribing clinicians. For these interactions, it should be considered to omit the overrule by decreasing the severity score and/or by reassessing and reformulating the time interval of the defined interaction in CDSS.

Second, the acceptance rate of the automatically electronic notes suggesting a switch from an IV to oral formulation of a bio-equivalent drug is relatively low: only $33 \%$ of the automated notes led to a therapy switch. Therefore, the content of the alert criteria and the validity of the automatic screening algorithm for sequential therapy should be evaluated.

Additionally, for the four selected domains, a total acceptance rate of $69 \%$ was observed. This in in line with the ADEAS study, where 128 (63\%) out of 204 actions led to a documented modification in therapy [23].

However, for the alerts for which only an electronic notes was sent for the four selected domains, a moderate acceptance level of $56 \%$ was seen. This implies that almost half of the advices was not accepted or perhaps not read by the physician. Along with the reasons mentioned by clinicians to deny the advice, the clinical relevance of clinical rules for which an advice is usually not accepted needs to be assessed. In the near future, a satisfaction survey is planned for physicians to evaluate their general experiences with this service, their overall reasons for agreeing or disagreeing with the pharmacotherapeutic advice and their specific wishes or comments for future expansion.

In contrast, the majority of advices given by a phone call on top of the electronic note was accepted (83\%). This was expected since a phone call is only performed for a medication order with a very high risk of ADE or DRP. It also indicates that an additional phone call may have a greater impact on the physician with a higher acceptance rate. Therefore it should be considered whether a phone call should be carried out faster.

Third, the acceptance level may also have been overestimated which is inherent to the retrospective evaluation of the acceptance rate: in some cases the physician could have modified the prescription independently of the pharmacy alert. 
In conclusion, a number of benefits but also limitations were identified aiming for the optimization of CMA. Next to further improving the CMA (focusing on increasing the specificity and reconsidering the basic CDSS for DDIs), an expansion of the service is planned to cover a much wider range of drugs or drug classes and to cover more patient groups with the aim of avoiding even more preventable ADEs. First, clinical rules focusing on a specific pharmacotherapeutic domain will be developed, i.e. focusing on antimicrobial stewardship, anticoagulation therapy, postoperative pain management and total parenteral nutrition therapy. Second, as it has been described in literature that critically ill patients are more prone to suffer from ADEs, an ICU-focused implementation of the CMA service is also planned. Lastly, future research (i.e. an interrupted time series analysis) is foreseen to measure the additional effect of the advanced clinical rules on top of basic CPOE/ CDSS and bedside clinical pharmacy in preventing PIMs in a controlled design. After this analysis, we need to reconsider if some alerts might be better situated as CDSS warnings to support physicians at the moment of prescribing in case immediately action is necessary.

\section{Conclusion}

CMA involves trained hospital pharmacists who daily check high risk prescriptions generated by advanced clinical rules integrating patient specific characteristics with information on medication. This service is valuable as it yielded a high number of pharmacists' actions for clinically relevant alerts with an adequate acceptance rate by physicians. In our opinion, CMA could support medication surveillance and optimize pharmacotherapy in synergy with already integrated basic CPOE/CDSS and bedside clinical pharmacy. Otherwise, the study also highlighted a number of limitations, allowing further research and improvement of the service.

\section{Additional file}

Additional file 1: Definite set of 78 advanced clinical rules. (DOCX $16 \mathrm{~kb}$ )

\begin{abstract}
Abbreviations
ADE: Adverse drug event; CDSS: Clinical Decision Support System; CMA: Check of Medication Appropriateness; CPOE: Computerized Physician Order Entry; DDI: Drug-drug interaction; DOAC: Direct oral anticoagulant; DRP: Drug-related problem; FTE: Full-time equivalent; HIS: Hospital Information System; ICU: Intensive care unit; IV: Intravenous; LMWH: Low molecular weight heparin; P\&T: Pharmacy \& Therapeutics; PIM: Potentially inappropriate medication
\end{abstract}

\section{Acknowledgements}

The authors would like to thank: Sabrina De Winter for the help and insightful comments during the development of the CMA service; and Krista D'Hondt for her assistance in data analysis.

Permission for acknowledgements was obtained.

\section{Funding}

The study was not funded.
Availability of data and materials

The datasets used and/or analysed during the current study are available from the corresponding author on reasonable request.

\section{Author's contributions}

$C Q$, TDR and IS designed the study and performed analysis, writing and revision of the manuscript. CQ and TVN implemented the study. WEP, BVDB, SS and MC did supervision and reviewed the manuscript. All authors approved their submission for publication.

\section{Ethics approval and consent to participate}

The Ethics Committee Research University Hospitals Leuven, Belgium (OG032) approved this study (S61615).

The need for informed consent was waived by the Clinical Trial Centre of the University Hospitals Leuven as this quality-improvement study was set up as standard practice. Advices will be provided by the clinical pharmacist directly the treating physician, who will finally decide on treatment adaptation. Each hospitalized patient is eligible for inclusion in the CMA service. No exclusion criteria are defined. Patients will not provide informed consent as this service will be implemented as standard of care.

\section{Consent for publication}

Not applicable.

\section{Competing interests}

All authors declare that they have no competing interests.

\section{Publisher's Note}

Springer Nature remains neutral with regard to jurisdictional claims in published maps and institutional affiliations.

\section{Author details}

'Department of Pharmaceutical and Pharmacological Sciences, KU Leuven, Herestraat 49, B-3000 Leuven, Belgium. ${ }^{2}$ Pharmacy Department, University Hospitals Leuven, Herestraat 49, B-3000 Leuven, Belgium. ${ }^{3}$ Department of Microbiology and Immunology, KU Leuven, Herestraat 49, B-3000 Leuven, Belgium. ${ }^{4}$ Department of General Internal Medicine, University Hospitals Leuven, Herestraat 49, B-3000 Leuven, Belgium. ${ }^{5}$ Department of Public Health and Primary Care, KU Leuven, Herestraat 49, B-3000 Leuven, Belgium. ${ }^{6}$ Department of Information Technology, University Hospitals Leuven, Herestraat 49, B-3000 Leuven, Belgium.

Received: 23 August 2018 Accepted: 22 January 2019

Published online: 11 February 2019

\section{References}

1. Roughead EE, Gilbert AL, Primrose JG, et al. Drug-related hospital admissions: a review of Australian studies published 1988-1996. Medical J Austr. 1998;168(8):405-8

2. Pedrós C, Quintana B, Rebolledo M, et al. Prevalence, risk factors and main features of adverse drug reactions leading to hospital admission. Eur J Clin Pharmacol. 2014;70(3):361-7.

3. Leendertse AJ, Egberts AC, Stoker LJ, et al. Frequency of and risk factors for preventable medication-related hospital admissions in the Netherlands. Arch Intern Med. 2008;168(17):1890-6.

4. Guchelaar HJ, Colen HB, Kalmeijer MD, et al. Medication errors: hospital pharmacist perspective. Drugs. 2005;65(13):1735-46.

5. Frontini R, Miharija-Gala T, Sykora J. EAHP survey 2010 on hospital pharmacy in Europe: parts 4 and 5. Clinical services and patient safety. Eur J Hosp Pharm. 2013;20:69-73.

6. Kaboli PJ, Hoth AB, McClimon BJ, et al. Clinical pharmacists and inpatient medical care: a systematic review. Arch Intern Med. 2006;166:955-64.

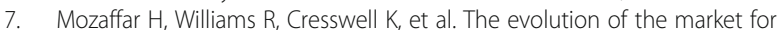
commercial computerized physician order entry and computerized decision support systems for prescribing. J Am Med Inform Assoc. 2016;23:349-55.

8. Shortliffe EH. Computer programs to support clinical decision making JAMA. 1987;258(1):61-6.

9. Kuperman GJ, Bobb A, Thomas H, et al. Medication-related clinical decision support in computerized provider order entry systems: a review. J Am Med Inform Assoc. 2007;14(1):29-40. 
10. Rommers MK, Teepe-Twiss IM, Guchelaar HJ. A computerized adverse drug event alerting system using clinical rules: a retrospective and prospective comparison with conventional medication surveillance in the Netherlands. Drug Saf. 2011:34(3):233-42.

11. de Wit HAJM, Mestres Gonzalvo C, Cardenas J, et al. Evaluation of clinical rules in a standalone pharmacy based clinical decision support system for hospitalized and nursing home patients. Int J Med Inform. 2015;84(6):396-405.

12. de Wit HAJM, Hurkens KPGM, Mestres Gonzalvo C, et al. The support of medication review in hospitalised patients by a clinical decision support system. Springerplus. 2016;5(1):871.

13. Eppenga WL, Derijks HJ, Conemans JM, et al. Comparison of a basic and an advanced pharmacotherapy-related clinical decision support system in a hospital care setting in the Netherlands. J Am Med Inform Assoc. 2012;19(1):66-71.

14. Ammenwerth E, Schnell-Inderst P, Machan C, et al. The effect of electronic prescribing on medication errors and adverse drug events: a systematic review. J Am Med Inform Assoc. 2008;15(5):585-600.

15. Eslami S, de Keizer NF, Abu-Hanna A. The impact of computerized physician medication order entry in hospitalized patients: a systematic review. Int J Med Inform. 2008;7(6):365-76.

16. Jaspers MW, Smeulers M, Vermeulen $\mathrm{H}$, et al. Effects of clinical decisionsupport systems on practitioner performance and patient outcomes: a synthesis of high-quality systematic review findings. J Am Med Inform Assoc. 2011;18(3):327-34

17. Pearson SA, Moxey A, Robertson J, et al. Do computerised clinical decision support systems for prescribing change practice? A systematic review of the literature. BMC Health Serv Res. 2009:9:154

18. van Doormaal JE, van den Bemt PM, Zaal RJ, et al. The influence that electronic prescribing has on medication errors and preventable adverse drug events: an interrupted time-series study. J Am Med Inform Assoc 2009:16(6):816-25.

19. Schedlbauer A, Prasad V, Mulvaney C, et al. What evidence supports the use of computerized alerts and prompts to improve clinicians' prescribing behavior? J Am Med Inform Assoc. 2009;16:531-8.

20. van der Sijs H, Aarts J, Vulto A, et al. Overriding of drug safety alerts in computerized physician order entry. J Am Med Inform Assoc. 2006;13(2): $138-47$.

21. Mermel LA, Allon M, Bouza $\mathrm{E}$, et al. Clinical practice guidelines for the diagnosis and management of intravascular catheter-related infection: 2009 update by the Infectious Diseases Society of America. Clin Infect Dis. 2009;49(1):1-45

22. Heidbuchel $H$, Verhamme $P$, Alings $M$, et al. European heart rhythm association practical guide on the use of new oral anticoagulants in patients with non-valvular atrial fibrillation. Europace. 2013;15(5):625-51.

23. Rommers MK, Zwaveling J, Guchelaar HJ, et al. Evaluation of rule effectiveness and positive predictive value ofclinical rules in a Dutch clinical decision support system in dailyhospital pharmacy practice. Artif Intell Med. 2013;59:15-21.

24. Silverman JB, Stapinski CD, Huber C, et al. Computer-based system for preventing adverse drug events. Am J Health Syst Pharm. 2004;61:1599e603.

25. Jha AK, Laguette J, Seger A, et al. Can surveillance systems identify and avert adverse drug events? A prospective evaluation of a commercial application. J Am Med Inform Assoc. 2008;15:647-53.

26. Raschke RA, Gollihare B, Wunderlich TM, et al. A computer alert system to prevent injury from adverse drug events. JAMA. 1998;280(15):1317-21.

\section{Ready to submit your research? Choose BMC and benefit from:}

- fast, convenient online submission

- thorough peer review by experienced researchers in your field

- rapid publication on acceptance

- support for research data, including large and complex data types

- gold Open Access which fosters wider collaboration and increased citations

- maximum visibility for your research: over $100 \mathrm{M}$ website views per year

At BMC, research is always in progress.

Learn more biomedcentral.com/submissions 